\title{
An HIT Solution for Clinical Care and Disaster Planning: How One health Center in Joplin, MO Survived a Tornado and Avoided a Health Information Disaster
}

\author{
Peter Shin ${ }^{1,2}$, Feygele Jacobs ${ }^{2}$
}

${ }^{1}$ George Washington University

${ }^{2}$ RCHN Community Health Foundation

\section{Abstract}

Since taking office, President Obama has made substantial investments in promoting the diffusion of health information technology (IT). The objective of the national health IT program is, generally, to enable health care providers to better manage patient care through secure use and sharing of health information. Through the use of technologies including electronic health records, providers can better maintain patient care information and facilitate communication, often improving care outcomes. The recent tornado in Joplin, MO highlights the importance of health information technology in the health center context, and illustrates the importance of secure electronic health information systems as a crucial element of disaster and business continuity planning. This article examines the experience of a community health center in the aftermath of the major tornado that swept through the American Midwest in the spring of 2011, and provides insight into the planning for disaster survival and recovery as it relates to patient records and health center data.

Key words: health information technology; public health; emergency preparedness; data recovery; health centers; low-income communities

\section{Introduction}

The devastating impact on health care facilities of 2005's hurricanes Katrina and Rita served to underscore the importance of protecting critical patient information, and maintaining patient records in a manner consistent with ensuring continuity of care. The horrific events of September 11, 2001, exposed the limitations of existing reporting and communication systems, and highlighted the need to strengthen population surveillance and community health measurement mechanisms to facilitate both rapid detection and expedited response to public health emergencies and bioterrorism. Similarly, the tremendous loss of paper patient records 
during the catastrophic 2005 storms showed that many health systems were ill-prepared for disaster situations.[1] Faced with a natural disaster, many providers of all types including local hospitals, health centers and physician offices, lacked the infrastructure necessary to ensure ongoing access to patient profiles, medication histories, lab results, and other vital patient information.

Community health centers (CHCs), initiated as a Federal demonstration program approximately 45 years ago, have evolved into a core of the health care safety net for low-income and medically underserved populations. Today, over 1,200 health centers, with 8,000 locations, serve approximately 20 million low-income patients across the country in urban inner-city and isolated, rural communities.[2] CHCs are located in high-need areas with high rates of poverty, poor health indicators and limited provider resources, offer essential services in accessible locations including public housing projects and schools, and serve as critical source of care for designated special populations and transient families, such as homeless and migrant and seasonal farmworkers.[3] Additionally, more than 90 percent of health center patients nationally are lowincome, approximately two-third of patients are non-white and one-quarter of health center patients prefer services in languages other than English, and may require translation and language support.[4]

Over the course of the last several years, community health centers have embraced the use of information technology to manage patient records, facilitate inter- and intra-facility referrals, expedite public health and grant-related reporting, and measure health care and operational outcomes.[5] Since taking office in January 2009, President Obama has prioritized the diffusion of health information technology (HIT) and funded substantial HIT investments in the general health care provider community and in community health centers in particular.[6] The American Recovery and Reinvestment Act of 2009 (ARRA) and Affordable Care Act of 2010 (ACA) financially incentivize health centers to adopt and use health information technology, especially electronic health records. A recent survey conducted by the National Association of Community Health Centers shows that between 2006 and 2010, the proportion of all CHCs that transitioned from paper to fully electronic medical records increased from 13 percent to 45 percent.[7] Still, electronic capacity remains uneven, as does infrastructure agility to withstand disaster and ensure continuity for patient care. Based on discussions with health center leadership and staff of a multi-site community health center with administrative offices in Neosho, Missouri we describe the strategies used by the center to protect access to clinical and operational information systems, and identify the lessons learned and ongoing vulnerabilities that are important in evaluating and planning for any disaster scenario.

\section{Joplin, MO/Access Family Care}

On Sunday, May 22, 2011, a category EF-5 tornado (with at least 200 mile per hour winds) destroyed or damaged thousands of buildings and homes over a 15-17 mile long and threequarter mile area in Joplin, MO, resulting in an estimated 162 deaths and hundreds of non-fatal 
injuries.[8] The storm devastated the city, including its largest physician complex, regional community hospital, and many physician and dental offices, and affected the operations of all providers locally, including the area's established CHC. The U.S. Department of Health and Human Services (HHS) declared the area a public health disaster area and dispatched staff from the National Disaster Medical System (NDMS) to help assess the extent of the damage and begin an immediate assistance and recovery planning process.[9]

Access Family Care (AFC), founded in the mid -1990s as Ozark Tri-County Health Care, is a federally qualified health center serving the communities of southwest Missouri, including the Joplin area. AFC operates 4 sites, including two in the city of Joplin, and serves approximately 15,000 patients annually. The center provides a full range of comprehensive primary care medical and dental services for adults and children, as well as some specialty care. Approximately 98 percent of AFC's patients are low-income (i.e., have incomes at or below 200 percent of the federal poverty level); 46 percent have health insurance coverage through Medicaid and 40 percent are uninsured.[10]

While the tornado destroyed AFC's smallest site, a primary care and behavioral health satellite located in Joplin proper, the center was able to provide continuity of care for their registered patients across all sites as well as support community emergency relief efforts, as a result of their preparation, experience, and, in part, good fortune. AFC's ability to withstand the storm was determined in large part by early and effective information technology planning. As AFC planned for the transition from paper to electronic medical records (EMRs), they sought a mobile and accessible system that would render minimal disruption to an efficient workflow, while providing a secure operational platform. AFC concluded that to optimize efficiency, they would need to adopt a solution that would permit their providers to access the system from remote locations and use the EMR and other clinical application(s) from any secure computer. Finally, the health center wanted the flexibility to use both fixed and mobile devices, including iPads and PCs. After reviewing and evaluating various options, the center selected NeoTech Solutions, an experienced local IT consulting firm based in Joplin, MO, which offered a hosted solution including a "virtual desktop" environment to assist them with the HIT implementation process. NeoTech was able to offer creative and cost-effective options for the center, including smart-card technology, which allows providers to access software and applications from any terminal or device at the point they left off, without needing a repeat log-in. NeoTech was integral to the center's EMR adoption process and from the inception of the project three years ago, AFC's core IT systems and applications, including its EMR (GE Centricity) and electronic dental record (Dentrix), have been hosted through NeoTech and maintained at a secure off-site data center.

Thus, the ability for the center to function smoothly depends not only on its internal capacity and infrastructure, but on that of its key HIT partner. NeoTech's hosting capability was tested twice: first, by a fire in March 2010 that originated in a restaurant next door to their facility and later, by the May 2011 tornado. While NeoTech's physical facility was compromised during the fire, 
they successfully relocated the data center to a temporary location and were back in operation in only 48 hours; there was no data loss and AFC was able to regain full data access the following day. After the fire, NeoTech rebuilt their hosting facility and made significant structural improvements to the data center. However, when the tornado struck on Sunday May 22, just 14 months later, NeoTech's building was largely destroyed; only the north section of the building, which was reinforced to accommodate the data center, withstood the storm. Miraculously, no data was lost despite the destruction of the premises and surrounding property. NeoTech again relocated to temporary quarters in order to ensure that client service was not disrupted, and was able to resume operations in just 12 hours. This proved critical for the health center. When they reopened in the days immediately following the storm AFC became the focal service delivery point for their own patients, as well as an emergency access point for others in the community, providing emergency medical triage and medication refills for community residents in need, while continuing to manage direct care and assist their own staff and staff families who were affected by the storm. NeoTech helped the health center establish on-site intranet connectivity so that there was minimal disruption; loss of internet in the local area, however, lasted a week and prevented connecting to external resources. AFC leadership noted that the city and county performed effectively with respect to emergency preparedness and response activities, clearing roads within a few days and fully restoring power to the city within a week. However, with few health care providers in the area, and the local hospital destroyed, AFC became an essential part of the response and recovery team.

From AFC's perspective, the return on the investment of adopting electronic records and secure offsite hosting was invaluable, and allowed them to function optimally in a disaster scenario. The one-time EMR acquisition and installation cost was approximately 4 percent of the center's operating budget; an additional 1 percent was allocated for initial implementation and support of EMR adoption. The initial EMR installation lacked some key features, such as document scanning and E-prescribing software, which were subsequently added at a nominal cost. AFC also later incurred some additional costs to connect the EMR with a third-party lab. Two years after the initial installation, AFC added eight more EMR licenses. While the center incurred some additional costs after the initial installation, its operating budget grew and today, the proportion of the center's operating budget dedicated to annual EMR licensing and support remains at $1 \%$. The center also budgeted $1 \%$ for off-site hosting, plus EDI services and Eprescribing at approximately $0.6 \%$ of the total budget. The center reports that the value associated with the cost is immeasurable; it has allowed AFC to manage patient care effectively across multiple sites; improve efficiency, and withstand the effects of the tornado that devastated much of the community. NeoTech, for its part, elected not to rebuild an independent facility after the tornado, and has since moved to a secure co-location site that offers considerable resilience and ability to expand capacity. 


\section{Discussion}

While CHCs are major providers of primary health care to individuals, they also provide a critical link that connects lower income and vulnerable communities with initiatives aimed at promoting community-wide health improvement and reducing the incidence of disease and disability.[11] Because of their strategic locations, CHCs can play a critical role in communitywide surveillance, act as first responders in the event of a public health emergency, and provide continuous physical and mental health services in the event of a major disaster.[12] Indeed, CHCs have engaged in precisely this type of activity over the years, serving as part of the public health surveillance infrastructure in low-income communities. ${ }^{1}$ Additionally, many $\mathrm{CHCs}$ participate in city and county emergency readiness planning.[13] Health centers provide hospital back-up and non-critical care, offer vaccinations during outbreaks, serve as surveillance and detection sites for possible outbreaks, and furnish essential information and education to the community.

The Centers for Disease Control and Prevention (CDC-P) have identified several major categories of natural disasters and other catastrophic events for community- and provider-based emergency planning and response, and CHCs have an essential role in disaster preparedness. In reality, no single health center is equipped or staffed to independently address a large-magnitude natural or man-made disaster. However, as the backbone of the nation's primary care safety net system, the adoption by $\mathrm{CHCs}$ of a secure health information technology platform serves to better link lower-income and vulnerable communities with other providers to promote continuity of care and helps reduce the incidence of disease and disability, particularly in the wake of natural disasters.

The experience of Access Family Care in Joplin, as well as of other community health centers at the epicenter of recent disasters, underscores the importance of investment in HIT at both the health center and community level. The operating costs incurred by the center are a manageable share of the overall budget and allow for operational and programmatic efficiencies while supporting the delivery of care. In the wake of the recent tornado, these dollars are clearly money well spent.

\section{Recommendations}

AFC's ability to quickly recover patient medical records underscores both the significance of federal investments to bolster adoption of health information technology, and the importance of

${ }^{1}$ For example, through a cooperative agreement with the CDC, the National Association of Community Health Centers, Inc. launched the Adolescent and School Health Initiative in 1993 to significantly enhance the capacity of health centers to expand and improve preventive and primary health programs targeted to youth at high risk for HIV/AIDS, sexually transmitted infections, and other health problems through developing school-based or school-linked comprehensive services in cooperation with schools in their communities. 
continued investments. AFC was able to resume operations quickly because they had appropriate IT capacity in place, but their experience is not necessarily the norm. Today, HIT initiatives are focused largely on electronic capture of meaningful clinical data, the use of data to track and improve quality, and the exchange of patient information in a structured format. However, there is relatively little attention to how $\mathrm{CHCs}$ and other providers should better secure their data and prepare for possible interruptions to care and information access. Therefore, The Bureau of Primary Health Care, which oversees the Health Centers program, should develop clear and detailed guidance that can be employed by CHCs to help plan for and overcome disaster situations. Such guidance should include alternatives and recommendations, based on industry standards, for physical security, data back-up, redundancy planning, staff training, and examples of effective strategies used by CHCs.

Health centers should also be active partners in their community's emergency preparedness efforts. Although most communities rely on hospital resources in the event of disasters, CHCs should seek to be active partners in local preparedness planning, train staff in emergency response and prepare their facilities to serve as critical access points. Because health centers are often located in isolated communities and serve hard-to-reach populations, state, county, and local agencies must help to equip CHCs to participate as emergency responders, and facilitate the inclusion of $\mathrm{CHCs}$ in preparedness planning.

Finally, data security should be identified as a specific priority for funding support. Federal HIT funding presently supports acquisition and implementation, and offer quality incentives, but does not provide direct support for securing data. Many CHCs may be unable to fund optimal data or hosting solutions without specific support, especially as local and state health center funding continues to decline due to economic downturn. AFC's experience demonstrates that investment in a local, secure hosting center is not only cost effective, but essential for the continued provision of quality care, especially in areas at high risk for natural disasters.

\section{Corresponding Author}

Peter Shin

Associate Professor of Health Policy

Research Director, RCHN/ Geiger Gibson

Email: pshin@gwu.edu

\section{References}

1. National Association of Community Health Centers, Legacy of Disaster Health Centers and Hurricane Katrina. One Year Later, 2006. Available at: http://www.nachc.com/client/documents/ issues-advocacy/policy-library/research-data/research-reports/katrinareport.pdf (Accessed

September 16, 2011). 
2. 2010 Uniform Data System (UDS), Health Resources and Services Administration (HRSA) -all federally-funded health centers are required to submit annual reports on their financial and clinical performance along with patient profiles.

3. HRSA. 2011. Health Centers: Where to go for care you can afford. Available at http:// www.hrsa.gov/ourstories/healthcenter/healthcenterweek.html. (Accessed September 16, 2011) 4. Hawkins D, Groves D. 2011. The Future Role of Community Health Centers in a Changing Health Care Landscape. J Ambul Care Manage. 34(1), 90-99. http://dx.doi.org/10.1097/ JAC.0b013e3182047e87

5. Association of State and Territorial Health Officials, National Association of County and City Health Officials, and National Association of Community Health Centers, Collaborating with Community Health Centers for Preparedness. 2008. Available at http://www.nachc.com/client/ Collaborating with Community Health Centers for Preparedness.pdf (Accessed September 16, 2011)

6. Blumenthal D. 2009. Stimulating the Adoption of Health Information Technology. N Engl J Med. 360, 1477-79. http://dx.doi.org/10.1056/NEJMp0901592

7. 2006 Harvard University, George Washington University, and the National Association of Community Health Centers survey and the 2010-11 GW Geiger Gibson/RCHN Research Collaborative survey of health centers.

8. 2010 UDS, HRSA, provided by Access Family Care.

9. Office of the Assistant Secretary for Preparedness and Response, U.S. Department of Health and Human Services. (May 27, 2011). Situation Reports: 2011 Joplin, Missouri Tornadoes. Available at: http://www.phe.gov/emergency/news/sitreps/Pages/missouri-tornado2011.aspx (Accessed September 16, 2011)

10. Kevin Murphy (2011, September 14). Joplin tornado death toll rises to 162 . Reuters. 11. National Association of Community Health Centers. Partnerships between Federal Qualified Health Centers and Local Health Departments for Engaging in the Development of a Communitybased System of Care, October 2010. Available at: http://www.nachc.com/client/documents/ PartnershipsBetweenFederallyQualifiedHealthCentersAndLocalHealthDepartmentsforEngagingI nTheDevelopmentOfACommunityBasedSystemOfCareNACHCOctober2010.pdf (Accessed September 16, 2011)

12. Wood, KM., Community Health Centers: The Untapped Resource for Public Health and Medical Preparedness, Homeland Security Affairs, January 2009; V(1): 1-39.

13. HRSA PIN 2007-15: Health Center Emergency Management Program Expectations. Available at http://bphc.hrsa.gov/policiesregulations/policies/pin200715expectations.html (Accessed September 16, 2011) 